\title{
Relação entre sobrecarga de cuidadores informais e nível de independência
}

\author{
de idosos hospitalizados
}

\section{Relationship between the burden of informal caregivers and the level of independence of hospitalized elders}

\author{
Érica Midori Ikegami ${ }^{1}$, Jéssica Rodrigues de Almeida ${ }^{2}$, Lara Andrade Souza ${ }^{3}$, \\ Isabel Aparecida Porcatti de Walsh ${ }^{4}$, Suraya Gomes Novais Shimano ${ }^{5}$, \\ Lislei Jorge Patrizzi ${ }^{6}$
}

http://dx.doi.org/10.11606/issn.2238-6149.v29i2p129-134

\begin{abstract}
Ikegami EM, Almeida JR, Souza LA, Walsh IAP, Shimano SGN, Patrizzi LJ. S Relação entre sobrecarga de cuidadores informais e nível de independência de idosos hospitalizados. Rev Ter Ocup Univ São Paulo. 2018 maio-ago.;29(2):129-34.

RESUMO: O objetivo do presente estudo foi avaliar a sobrecarga do cuidador informal, o nível de independência de idosos hospitalizados e verificar a relação entre eles. Trata-se de um estudo quantitativo, transversal e descritivo, realizado por meio de entrevistas individuais junto a 40 cuidadores de idosos internados no setor de Clínica Médica de um hospital público em Uberaba-MG. Dos cuidadores foram coletados dados sociodemográficos, referentes ao cuidado e nível de sobrecarga (Zarit Burden Interview) e dos idosos, sexo, idade, diagnóstico e nível de independência (Índice de Barthel). Foram utilizadas estatísticas descritivas e o Coeficiente de Correlação de Pearson para verificar a correlação entre sobrecarga do cuidador e nível de independência do idoso $(\mathrm{p}<0,05)$. Predominaram cuidadores do sexo feminino, com 1-4 anos de estudo, com companheiro, filhos dos idosos, que recebiam ajuda de outras pessoas para cuidar e despendiam mais de 15 horas diárias ao cuidado. Foi identificada sobrecarga dos cuidadores de moderada a leve $(62,5 \%)$, idosos com dependência total $(47,5 \%)$ e relação moderada negativa entre a sobrecarga do cuidador e nível de independência do idoso $(r=-0,423 ; p=0,007)$. Espera-se que a investigação contribua para o desenvolvimento de ações para o cuidador nos hospitais, visando a prevenção da sobrecarga e suas repercussões.
\end{abstract}

Descritores: Cuidadores; Idoso fragilizado; Hospitalização; Efeitos psicossociais da doença.
Ikegami EM, Almeida JR, Souza LA, Walsh IAP, Shimano SGN, Patrizzi LJ. S Relationship between the burden of informal caregivers and the level of independence of hospitalized elders. Rev Ter Ocup Univ São Paulo. 2018 May-Aug.;29(2):129-34.

\begin{abstract}
The objective of the present study was to evaluate the burden upon informal caregivers, the level of independence of the hospitalized elders and to verify the relationship between them. It is a quantitative, cross sectional and descriptive study, conducted by individual interviews with the 40 caregivers of the elders hospitalized in the Medical Clinic of a public hospital in Uberaba-MG. From the caregivers were collected sociodemographic data, relating to the care, and the burden level (Zarit Burden Interview) and from the elders, gender, age, diagnosis and the level of independence (Barthel Index). Descriptive statistics and Pearson's Correlation Coefficient were used to verify the correlation between the caregivers' burden and level of independence of the elder $(\mathrm{p}<0.05)$. There was a predominance of female caregivers, with 1-4 years of study, with partners, children of the elders, who received help from other people to care for and spent more than 15 hours daily to care. Moderate to light caregivers' burden $(62.5 \%)$, elders with total dependence $(47.5 \%)$ and moderate and negative link was found between the caregivers' burden and the level of independence of the elder $(r=-0.423 ; \mathrm{p}=0.007)$. This investigation is expected to contribute for the development of actions for the caregiver in hospitals, aiming to prevent their burden and its consequences.
\end{abstract}

Keywords: Caregivers; Frail elderly; Hospitalization; Cost of illness.

Este artigo é parte do Trabalho de Conclusão de Curso do Programa de Residência Integrada Multiprofissional em Saúde, Área de Concentração - Saúde do Idoso: Atenção Integrada e Humanizada em Saúde, eixo específico de Fisioterapia - UFTM.

1. Fisioterapeuta, mestre em Atenção à Saúde pela Universidade Federal do Triângulo Mineiro, Uberaba, MG, Brasil. ORCID: https://orcid.org/00000002-7262-5881. Email: erica.ikegami@gmail.com.

2. Fisioterapeuta, mestre em Ciências da Reabilitação pela Universidade Federal de Minas Gerais, Belo Horizonte, MG, Brasil. ORCID: https://orcid. org/0000-0002-1635-0190. Email: almeidajessica10@hotmail.com.

3. Fisioterapeuta, doutoranda em Atenção à Saúde da Universidade Federal do Triângulo Mineiro, Uberaba, MG, Brasil. e-mail: lara.asouza@yahoo.com. br / ORCID: https://orcid.org/0000-0003-4235-1215

4. Fisioterapeuta, doutora em Fisioterapia pela UFSCar, Professora Associada do Departamento de Fisioterapia Aplicada da Universidade Federal do Triângulo Mineiro, Uberaba, MG, Brasil. ORCID: https://orcid.org/0000-0002-2317-1326. Email: ewalsh@terra.com.br

5. Fisioterapeuta, doutora em Ciências da Reabilitação pela USP, Professora Adjunta do Departamento de Fisioterapia Aplicada da Universidade Federal do Triângulo Mineiro, Uberaba, MG, Brasil. ORCID: https://orcid.org/0000-0002-9421-9227. Email: surayagnovais@gmail.com.

6. Fisioterapeuta, doutora em Ciências Médicas pela USP, Professora Associada do Departamento de Fisioterapia Aplicada da Universidade Federal do

Triângulo Mineiro, Uberaba, MG, Brasil. ORCID: https://orcid.org/0000-0002-3729-7675. Email: lispatrizzi@gmail.com.

Endereço para correspondência: Érica Midori Ikegami. Av. Domingos Camerlingo Caló, 1633. Vila São José. Ourinhos, SP, Brasil. CEP: $19901-711$. Email: erica.ikegami@gmail.com. 


\section{INTRODUÇÃO}

envelhecimento é um processo heterogêneo
que ocorre de maneira particular em cada indivíduo $^{1}$. Para uma parcela da população idosa, a combinação de um ou mais fatores físicos, ambientais, socioeconômicos, genéticos e de hábitos de vida podem causar alterações nas funções do corpo e dependência da família, comunidade e sistema de saúde, influenciando negativamente sua participação na sociedade ${ }^{2}$.

A perda ou diminuição da capacidade funcional do idoso se traduz em dificuldade e necessidade de auxílio para execução de tarefas cotidianas básicas ou de maior complexidade, demandando a presença de um cuidador para assumir tais atividades ${ }^{3}$. Nesse contexto, o cuidador é a pessoa responsável pelos cuidados prestados ao idoso em situação de doença ou dependência, na realização das atividades de vida diária, que pode ter ou não remuneração pela função e ser ou não membro da família ${ }^{4}$.

A literatura tem destacado o papel e importância dos cuidadores nos hospitais ${ }^{5,6}$, ambiente que tem concentrado expressivo número de idosos com incapacidades e doenças complexas e persistentes ${ }^{7}$. A importância do cuidador é enfatizada pela Portaria $n^{\circ} 280$, de 7 de abril de $1999^{8}$, que afirma o papel dos hospitais de assegurarem a permanência dos familiares durante o período de internação, visto que sua presença está associada à melhoria da qualidade de vida dos pacientes ${ }^{8}$.

Estudos mostram que durante o período de internação hospitalar do idoso, o cuidado geralmente é assumido pelo cuidador informal, representado por um membro da família ${ }^{9,10}$. Cuidar do idoso hospitalizado implica em responsabilidades e disponibilidade de tempo, além de condicionar mudanças nos aspectos econômicos, físicos e/ou emocionais da vida do cuidador, tornando-o mais vulnerável a sobrecarga e a doença ${ }^{1}$.

A sobrecarga é caracterizada pelo esgotamento mental e físico, devido ao acúmulo de estressores, e se produz quando o cuidador se encontra desprovido de estratégias adequadas de enfrentamento para se adaptar a uma nova situação ${ }^{11}$, por exemplo, a internação hospitalar do idoso.

O cuidador é exposto a múltiplos fatores que favorecem a sobrecarga ${ }^{12}$, como o despreparo para o ato de cuidar $^{13,14}$, a insuficiência de recursos financeiros e de apoio da comunidade ${ }^{9}$, a adaptação a nova rotina ${ }^{1}$ e o nível de dependência da pessoa cuidada ${ }^{13,15}$. Destaca-se que prestar cuidado a um idoso em situação de dependência envolve renúncia do cuidador de suas relações interpessoais e exige dedicação e conhecimento ${ }^{16}$, que variam de acordo com as especificidades da doença ou a causa da incapacidade do idoso a ser cuidado ${ }^{12}$.

No hospital, embora os cuidados sejam providos por uma equipe técnica, o cuidador também acaba participando das rotinas de cuidado ${ }^{1}$, muitas vezes sem ter recebido treinamento ${ }^{13}$, resultando em práticas inseguras que podem comprometer a saúde do idoso e gerar sentimentos negativos de culpa, inutilidade e fracasso no cuidador ${ }^{6}$.

A sobrecarga do cuidador de idosos dependentes é abordada na literatura, porém, em outros contextos, como o domiciliar ${ }^{13,14}$. Estudos que verificaram a relação entre sobrecarga e dependência do idoso nos hospitais ainda são escassos e por isso necessitam de mais investigações, que podem contribuir para o estabelecimento de ações específicas para o cuidador.

Nessa perspectiva o objetivo do estudo foi avaliar a sobrecarga do cuidador informal, o nível de independência do idoso hospitalizado e verificar a relação entre eles.

\section{PROCEDIMENTOS METODOLÓGICOS}

Trata-se de um estudo de abordagem quantitativa, transversal e descritivo, realizado por meio de entrevista individual, no setor de Clínica Médica de um hospital público, localizado no município de Uberaba-MG. Os participantes foram abordados de julho a outubro de 2015, após atender os critérios de inclusão: ser cuidador informal de paciente idoso (com idade maior ou igual a 60 anos) internado por no mínimo 48 horas no setor, ter idade superior ou igual a 18 anos e possuir condições cognitivas avaliadas pelo Mini Exame do Estado Mental ${ }^{17}$, para responder aos questionários. Foram excluídos do estudo os cuidadores que não concordaram em responder a todos os instrumentos propostos.

Os cuidadores foram entrevistados durante a internação do idoso, em sala reservada nas dependências do hospital. Foi aplicado inicialmente roteiro de questões para obtenção das seguintes características do cuidador: sexo, idade, estado civil, escolaridade em anos de estudo, grau de parentesco com o idoso, se recebe ajuda de outras pessoas para cuidar do idoso e horas diárias dedicadas ao cuidado durante o período de internação hospitalar.

A sobrecarga do cuidador foi verificada por meio da escala Zarit Burden Interview (ZBI), traduzida e validada para a cultura brasileira por Scazfuca ${ }^{18}$. O escore varia de 0 a 88 pontos, onde pontuações mais altas se referem a maiores níveis de sobrecarga ${ }^{18}$. Foram utilizados os seguintes pontos de corte para classificação da sobrecarga: entre 61 e 88 pontos $=$ intensa; entre 41 e 60 pontos $=$ moderada a severa; entre 21 e $40=$ moderada a leve; e menos do que 21 pontos 
$=$ ausência de sobrecarga ${ }^{19}$.

Foram consultados os prontuários dos idosos para obtenção dos dados referentes a dias de internação, sexo, idade e diagnóstico do paciente. O nível de independência do idoso foi avaliado por meio do Índice de Barthel, validado no Brasil ${ }^{20}$. O instrumento avalia atividades de vida diária relacionadas ao cuidado pessoal, mobilidade, locomoção e eliminações. A pontuação varia de 0 a 100 pontos, com intervalos de cinco pontos, onde as maiores pontuações são condizentes com maior nível de independência ${ }^{20}$. Adotou-se a seguinte pontuação para classificação do nível de independência: $0-20=$ dependência total; $21-60=$ dependência severa; $61-90=$ dependência moderada; $91-99$ $=$ dependência leve; e $100=$ independência ${ }^{21}$.

Os dados foram tabulados no programa Microsoft Excel 2007 e as análises foram efetuadas com o auxílio do software Statistical Package for the Social Sciences (SPSS), versão 20.0. A análise de dados foi realizada por meio de estatísticas descritivas, utilizando-se frequências absolutas e relativas e medidas de tendência central e de variabilidade.

Após verificação da normalidade de distribuição da amostra pelo teste de Kolmogorov-Smirnov, a possível correlação entre a sobrecarga do cuidador e o nível de independência do idoso foi avaliada pelo Coeficiente de Correlação de Pearson. A interpretação da magnitude das correlações considerou a seguinte classificação dos coeficientes: fraca $(<0,3)$; moderada $(0,3$ a 0,59$)$; forte $(0,6$ a 0,9$)$ e perfeita $(1,0)^{22}$. Adotou-se nível de significância de $\mathrm{p}<0,05$.

O estudo foi conduzido após aprovação da pesquisa pelo Comitê de Ética e Pesquisa com Seres Humanos da Universidade Federal do Triângulo Mineiro sob o parecer $\mathrm{n}^{\circ}$ 1.121.346, CAAE $\mathrm{n}^{\circ}$ 45858915.9.0000.5154. Os cuidadores e idosos foram esclarecidos sobre os objetivos da pesquisa concordaram em participar do estudo mediante assinatura do Termo de Consentimento Livre e Esclarecido.

\section{RESULTADOS}

No total foram abordados 52 indivíduos no período, porém 12 não compuseram a amostra final, pois 2 tinham idade inferior a 18 anos; 3 não concordaram em responder todas as perguntas; 5 não eram cuidadores (apenas visitantes) e 2 não obtiveram pontuação satisfatória no Mini Exame do Estado Mental. Portanto, a amostra final foi composta por 40 cuidadores informais de 40 idosos internados no setor.

Os cuidadores eram indivíduos com média de idade de 53,25 $\pm 11,89$ anos e segundo a Tabela 1, houve predomínio do sexo feminino (87,5\%), com 1-4 anos de estudo (47,5\%), com companheiro $(72,5 \%)$ e filhos dos idosos (60\%). Verificou-se que $80 \%$ dos cuidadores recebiam ajuda de outra pessoa para cuidar do idoso e 57,5\% despediam mais de 15 horas diárias de cuidado durante $o$ período de internação hospitalar.

Tabela 1 - Características dos cuidadores informais de idosos internados no setor de Clínica Médica, Uberaba-MG, 2015

\begin{tabular}{|c|c|c|}
\hline Características dos cuidadores & $\mathbf{n}$ & $\%$ \\
\hline \multicolumn{3}{|l|}{ Sexo } \\
\hline Masculino & 5 & 12,5 \\
\hline Feminino & 35 & 87,5 \\
\hline \multicolumn{3}{|l|}{ Estado Civil } \\
\hline Com companheiro (a) & 29 & 72,5 \\
\hline Sem companheiro (a) & 11 & 27,5 \\
\hline \multicolumn{3}{|l|}{ Escolaridade } \\
\hline Analfabeto & 2 & 5 \\
\hline $1-4$ anos & 19 & 47,5 \\
\hline $5-8$ anos & 2 & 5 \\
\hline $9-12$ anos & 12 & 30 \\
\hline 13 anos ou mais & 5 & 12,5 \\
\hline \multicolumn{3}{|l|}{ Grau de parentesco com o idoso } \\
\hline Filho (a) & 24 & 60 \\
\hline Esposo (a) & 6 & 15 \\
\hline Genro (nora) & 2 & 5 \\
\hline Irmão (ã) & 5 & 12,5 \\
\hline Outros & 3 & 7,5 \\
\hline \multicolumn{3}{|l|}{$\begin{array}{l}\text { Recebe ajuda de outra pessoa no } \\
\text { cuidado }\end{array}$} \\
\hline Não & 8 & 20 \\
\hline Sim & 32 & 80 \\
\hline \multicolumn{3}{|l|}{$\begin{array}{l}\text { Horas diárias dedicadas ao } \\
\text { cuidado }\end{array}$} \\
\hline Menos do que $5 \mathrm{~h}$ & 2 & 5 \\
\hline De 5 a $10 \mathrm{~h}$ & 9 & 22,5 \\
\hline De 11 a $15 \mathrm{~h}$ & 6 & 15 \\
\hline Mais do que $15 \mathrm{~h}$ & 23 & 57,5 \\
\hline
\end{tabular}

Fonte: Das autoras, 2015.

Em relação às características dos idosos, houve predomínio do sexo masculino (60\%), com média de idade de $74,8 \pm 8,35$ anos, internados no setor por doenças do aparelho circulatório (53\%).

A média do escore de independência funcional dos idosos segundo o Índice de Barthel foi de 39,5 $\pm 38,6$. No que se refere à classificação, a Tabela 2 mostra o predomínio de idosos com dependência total $(47,5 \%)$ e considerando 
o conjunto dos participantes, $87,5 \%$ apresentavam algum nível de dependência.

Tabela 2 - Classificação do nível de independência do idoso, Uberaba, MG, 2015

\begin{tabular}{lcc}
\hline $\begin{array}{l}\text { Classificação da independência do } \\
\text { idoso }\end{array}$ & n & \% \\
\hline Dependência total & 19 & 47,5 \\
Dependência severa & 9 & 22,5 \\
Dependência moderada & 3 & 7,5 \\
Dependência leve & 4 & 10 \\
Independência & 5 & 12,5 \\
\hline
\end{tabular}

Fonte: Das autoras, 2015.

$\mathrm{Na}$ avaliação do nível de sobrecarga do cuidador por meio da ZBI verificou-se que a média de sobrecarga

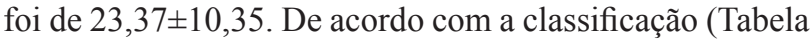
$3)$, ocorreu predomínio de cuidadores com sobrecarga de moderada a leve $(62,5 \%)$. Quanto à investigação da relação entre a sobrecarga do cuidador e o nível de independência do idoso hospitalizado foi constatada correlação moderada negativa, estatisticamente significante $(r=-0,423$ e $p=0,007)$, ou seja, quanto menor a sobrecarga, maior o nível de independência do idoso.

Tabela 3 - Classificação do nível de sobrecarga do cuidador, Uberaba-MG, 2015

\begin{tabular}{lcc}
\hline $\begin{array}{l}\text { Classificação da sobrecarga do } \\
\text { cuidador }\end{array}$ & n & \% \\
\hline $\begin{array}{l}\text { Sobrecarga severa a moderada } \\
\text { (entre 41 a } 60 \text { pontos) }\end{array}$ & 2 & 5,0 \\
$\begin{array}{l}\text { Sobrecarga moderada a leve } \\
\text { (entre 21 a 40 pontos) }\end{array}$ & 25 & 62,5 \\
$\begin{array}{l}\text { Ausência de sobrecarga } \\
(<20 \text { pontos) }\end{array}$ & 13 & 32,5 \\
\hline
\end{tabular}

Fonte: Das autoras, 2015.

\section{DISCUSSÃO}

Concernente às características dos cuidadores informais dos idosos, o predomínio de mulheres e filhos (as) assumindo o cuidado do idoso hospitalizado corrobora com outro estudo realizado no contexto hospitalar ${ }^{5}$. Os achados confirmam o papel sociocultural atribuído tanto à figura feminina, na realização dos afazeres domésticos e no cuidado prestado à família ${ }^{16}$, quanto dos filhos, como forma de retribuição pelos cuidados recebidos de seus pais durante a vida ${ }^{1}$.

A média de idade $(53,25 \pm 11,89$ anos $)$ e o estado civil dos cuidadores desse estudo foram semelhantes aos encontrados na literatura ${ }^{10,23}$. Quanto à escolaridade foram identificados níveis mais baixos, corroborando com estudo que indica que indivíduos com menor formação tendem a assumir a tarefa de cuidar e os serviços domésticos, visto que o mercado de trabalho formal exige maiores níveis de escolaridade ${ }^{13}$.

Embora $80 \%$ dos cuidadores tenham relatado que recebem ajuda de outra pessoa no cuidado prestado ao idoso, a maioria ainda despende mais de 15 horas diárias de cuidado no hospital, o que condiz com achados na literatura ${ }^{5}$. Atribuir o cuidado a um único membro da família também é comum no âmbito domiciliar, o que pode gerar estresse no cuidador, visto que na maioria das vezes o familiar não está preparado para assumir a função imposta ${ }^{12}$.

Quanto ao idoso que recebe o cuidado, embora as mulheres se destaquem na literatura como dependentes e necessitando de cuidados nos hospitais ${ }^{11,24}$, neste estudo houve predomínio do sexo masculino, corroborando com alguns estudos prévios ${ }^{5,23}$, o que pode ser justificado pelo nível de cuidado insatisfatório que o homem tem com a sua saúde, favorecendo a ocorrência de internações hospitalares ${ }^{25}$.

A média de idade relativamente alta dos idosos $(74,8 \pm 8,35$ anos) também foi constatada em outros estudos $^{5,25}$ e pode ser justificada pelo aumento da expectativa de vida da população idosa no Brasil, marcada por quadros de incapacidade, doenças crônicas e internações hospitalares ${ }^{7}$. A causa de internação dos idosos desse estudo condiz com levantamento realizado, em que as doenças do aparelho circulatório se destacaram como causa entre os anos de 2003 a $2012^{24}$.

Segundo o Índice de Barthel, o predomínio de idosos com algum nível de dependência, corrobora com estudo realizado no âmbito hospitalar ${ }^{11}$. O envelhecimento é marcado pelas doenças crônicas e suas repercussões como a dependência, o que representa um desafio para a saúde pública, visto que as projeções apontam a continuidade desse fenômeno nos próximos anos?

A sobrecarga de moderada a leve identificada entre os cuidadores se assemelha aos achados de estudo que investigou a prevalência da sobrecarga de cuidadores familiares de idosos com sequela de acidente vascular encefálico ${ }^{14}$, porém, em âmbito domiciliar. A relação entre sobrecarga do cuidador e nível de independência do idoso está bastante consolidada na literatura ${ }^{13,15,16}$, entretanto nos hospitais as investigações ainda são escassas, indicando necessidade de estudos no Brasil, nesses locais. Segundo revisão integrativa, a exigência e complexidade do cuidado se intensificam de acordo com a dependência da 
pessoa cuidada, favorecendo o estresse físico e mental dos cuidadores $^{12}$.

A classificação da sobrecarga predominante entre os cuidadores e sua relação moderada com o nível de independência do idoso pode ser justificada neste estudo, pelo fato de $80 \%$ dos cuidadores receberem ajuda de outra pessoa no cuidado com o idoso. Além do cuidar não ser solitário, segundo Cabral e Nunes ${ }^{5}$, nos hospitais os cuidadores recebem supervisão e/ou treinamento da equipe de profissionais, o que fornece maior segurança, reduzindo o nível de sobrecarga quando comparado ao cuidado que prestam no domicílio. Ademais, Pavin e Carlos ${ }^{1}$ afirmam que embora o ato de cuidar possa gerar sobrecarga e influenciar negativamente a qualidade de vida do cuidador, existem as influências positivas do ato de cuidar como os sentimentos de amor e retribuição, principalmente por parte dos filhos(as).

Ainda que predomine sobrecarga de moderada a leve entre os cuidadores entrevistados e relação ainda moderada entre a sobrecarga desses indivíduos e o nível de independência dos familiares idosos hospitalizados; $67,5 \%$ dos entrevistados já apresentam algum nível de sobrecarga, que em longo prazo pode se intensificar. Segundo Nardi et al..$^{15}$, as tarefas relacionadas ao cuidado não são estáveis, ou seja, podem sofrer modificações com o passar do tempo, à medida que aumenta a debilidade e dependência do idoso, demandando maiores esforços do cuidador para suprir as necessidades da pessoa cuidada.

Cabe ressaltar que na maioria das vezes, o cuidador se depara com a função de cuidar do idoso sem treinamento prévio e desprovido de informações, o que o obriga a assimilar conhecimentos com a prática ${ }^{13}$. Nesse contexto destaca-se a importância de facilitadores do cuidado, como o apoio institucional e o bom relacionamento com as equipes de saúde ${ }^{10}$, que devem fornecer orientações durante a internação, para que os cuidadores sejam capazes de dar continuidade nos cuidados fora do ambiente hospitalar'.

O estudo apresentou algumas limitações como o delineamento transversal que não permitiu o estabelecimento de relações de causa e efeito; o número limitado de cuidadores que participaram e o alcance do estudo, cujos achados referem-se a um único hospital. Porém, destaca-se que os resultados podem orientar profissionais no subsídio de ações voltadas para a prevenção da sobrecarga do cuidador durante o período de internação do idoso.

Sugere-se que novos estudos sobre a temática sejam conduzidos, como os de delineamento longitudinal com amostras representativas, investigação de outros fatores que podem influenciar a sobrecarga nesse contexto e de estratégias de prevenção e enfrentamento.

\section{CONCLUSÃO}

O presente estudo constatou nível de sobrecarga de moderado a leve nos cuidadores, idosos com nível de dependência total e relação moderada negativa entre a sobrecarga do cuidador informal e o nível de independência do idoso hospitalizado. Considerando que essa associação no contexto hospitalar ainda é pouco explorada na literatura, espera-se que os resultados desse estudo forneçam bases para a condução de novas investigações sobre a temática, contribuindo para a criação de programas e ações específicas que objetivem prevenir a sobrecarga do cuidador durante o acompanhamento do idoso no contexto hospitalar.

Contribuições dos autores: Ikegami EM, participou de todas as etapas de elaboração do artigo (concepção, delineamento, coleta, organização e análise dos dados, redação e revisão). Almeida JR, auxiliou na coleta e organização dos dados. Souza LA, contribuiu na redação do artigo. Walsh IAP, contribuiu na análise dos dados e revisão crítica do artigo. Shimano GN e Patrizzi LJ, auxiliaram na revisão crítica e adequação do artigo. Todos os autores aprovaram a versão final do texto.

\section{REFERENCIAS}

1. Pavin RS, Carlos SA. A qualidade de vida de cuidadores informais de idosos hospitalizados. Rev Bras Ciênc Envelhecimento Hum. 2013;10(3):242-55. http://dx.doi. org/10.5335/rbceh.2013.3247.

2. Sudré MRS, Reiners AAO, Nakagawa JTT, Azevedo RCS, Floriano LA, Morita LHM. Prevalência de dependência em idosos e fatores de risco associados. Acta Paul Enferm. 2012;25(6):947-53. http://dx.doi.org/10.1590/S010321002012000600019.

3. Alves LC, Leite IC, Machado CJ. Conceituando e mensurando a incapacidade funcional da população idosa: uma revisão de literatura. Ciênc Saúde Colet. 2008;13(4):1199-207. http:// dx.doi.org/10.1590/S1413-81232008000400016

4. Brasil. Ministério da Saúde. Portaria n. 1395, de 9 de dezembro de 1999. Aprova a Política Nacional de Saúde do Idoso e dá outras providências. Diário Oficial da União, Brasília, n.237-E, p.20-24, 13 dez. 1999. Seção 1. [citado 29 jan. 2017]. Disponível em: http://crn3.org.br/Areas/Admin/Content/ upload/file-0711201573034.pdf

5. Cabral BPAL, Nunes CMP. Percepções do cuidador familiar 
Ikegami EM, et al. Relação entre sobrecarga de cuidadores informais. Rev Ter Ocup Univ São Paulo. 2018 maio-ago.;29(2):129-34.

sobre o cuidado prestado ao idoso hospitalizado. Rev Ter Ocup Univ São Paulo. 2015;26(1):118-27. http://dx.doi. org/10.11606/issn.2238-6149.v26i1p118-127.

6. Nayeri ND, Gholizadeh L, Mohammadi E, Yazdi K. Family Involvement in the Care of Hospitalized Elderly Patients. J Appl Gerontol. 2015;34(6):779-96. http://dx.doi. org/10.1177/0733464813483211.

7. Veras R. Envelhecimento populacional contemporâneo: demandas, desafios e inovações. Rev Saúde Pública. 2009;43(3):548-54. http://dx.doi.org/10.1590/S003489102009005000025 .

8. Brasil. Ministério da Saúde. Portaria n. 280, de 7 de abril de 1999. Torna obrigatório nos hospitais públicos, contratados ou conveniados com o Sistema Único de Saúde - SUS, a viabilização de meios que permitam a presença do acompanhante de pacientes maiores de 60 (sessenta) anos de idade, quando internados. Diário Oficial da União, Brasília, DF. n.66-E, 8 abr. 1999. Seção 1:14 [citado 15 jan. 2017]. Disponível em: http://bvsms.saude.gov.br/bvs/saudelegis/ gm/1999/prt0280_07_04_1999.html.

9. Dallalana TM, Batista MGR. Qualidade de vida do cuidador durante internação da pessoa cuidada em Unidade de Urgência/ Emergência: alguns fatores associados. Ciênc Saúde Coletiva. 2014;19(11):4587-94. http://dx.doi.org/ 10.1590/1413812320141911.16592013.

10. Chernicharo IM, Ferreira MA. Meanings of care for the hospitalized elderly from the perspective of caregivers. Esc Anna Nery. 2015;19(1):80-5. http://dx.doi.org/10.5935/14148145.20150011.

11. Muñoz-Campos NM, Lozano-Nuevo JJ, Huerta-Ramírez S, Escobedo-de la Cruz RC, Torres-Reyes P, Loaiza-Félix J. Asociación entre colapso del cuidador y pronóstico de pacientes geriátricos hospitalizados en el servicio de Medicina Interna. Med Int Mex. 2015;31 (5):328-35. http://www. medigraphic.com/pdfs/medintmex/mim-2015/mim155e.pdf

12. Baptista BO, Beuter M, Girardon-Perlini NM, Brondani CM, Budó ML, Santos NO. A sobrecarga do familiar cuidador no âmbito domiciliar: uma revisão integrativa da literatura. Rev Gaúc Enferm. 2012;33(1):147-56. http://dx.doi.org/10.1590/ S1983-14472012000100020.

13. Gratão ACM, Talmelli LFS, Figueiredo LC, Rosset I, Freitas CP, Rodrigues RAP. Dependência funcional de idosos e a sobrecarga do cuidador. Rev Esc Enferm USP. 2013;47(1):13744. http://dx.doi.org/10.1590/S0080-62342013000100017.

14. Costa TF, Costa KNFM, Martins K, Fernandes MGM, Brito SS. Burden over family caregivers of elderly people with stroke. Esc Anna Nery. 2015;19(2). http://dx.doi.

Recebido em: 12.07.2018

Aceito em: 03.10.2018 org/10.5935/1414-8145.20150048.

15. Nardi EFR, Sawada NO, Santos JLF. Associação entre a incapacidade funcional do idoso e a sobrecarga do cuidador familiar. Rev. Latino-Am. Enfermagem 2013;21(5):1096-103. http://dx.doi.org/10.1590/S0104-11692013000500012.

16. Fuhrmann AC, Bierhals CCBK, Santos NO, Paskulin LMG. Association between the functional capacity of dependant elderly people and the burden of family caregivers. Rev Gaúcha Enferm. 2015;36(1):14-20. http://dx.doi.org/10.1590/19831447.2015.01.49163.

17. Brucki SMD, Nitrini R, Caramelli P, Bertolucci PHF, Okamoto IH. Sugestões para o uso do mini-exame do estado mental no Brasil. Arq Neuro-Psiquiatr. 2003;61(3B):777-81. http:// dx.doi.org/10.1590/S0004-282X2003000500014.

18. Scazufca M. Brazilian version of the Burden Interview scale for the assessment of burden of care in carers of people with mental illnesses. Rev Bras Psiquiatr. 2002;24(1):12-7. http:// dx.doi.org/10.1590/S1516-44462002000100006.

19. Hébert R, Bravo G, Préville M. Reliability, Validity and Reference Values of the Zarit Burden interview for assessing informal caregivers of community-dwelling older persons with dementia. Can J Aging. 2000;19(4):494-507. https://doi. org/10.1017/S0714980800012484.

20. Minosso JSM, Amendola F, Alvarenga MRM, Oliveira MAC. Validação, no Brasil, do Índice de Barthel em idosos atendidos em ambulatórios. Acta Paul Enferm. 2010;23(2):218-23. http:// dx.doi.org/10.1590/S0103-21002010000200011.

21. Shah S, Vanclay F, Cooper B. Improving the sensitivity of the Barthel Index for stroke rehabilitation. J Clin Epidemiol. 1989;42(8):703-9. http://dx.doi.org/10.1016/08954356(89)90065-6.

22. Levin J, Fox JA. Estatística para ciências humanas. 9a ed. São Paulo: Prentice-Hall; 2004.

23. Souza ICP, Silva AG, Quirino ACS, Neves MS, Moreira LR. Perfil de pacientes dependentes hospitalizados e cuidadores familiares: conhecimento e preparo para as práticas do cuidado domiciliar. Rev Min Enferm. 2014;18(1):164-72. http://dx.doi. org/10.5935/1415-2762.20140013.

24. Marques LP, Confortin SC. Doenças do aparelho circulatório: principal causa de internações de idosos no Brasil entre 2003 e 2012. Rev Bras Ciênc Saúde. 2015;19(2):83-90. doi: http:// dx.doi.org/10.4034/RBCS.2015.19.02.01.

25. Storti LB, Fabrício-Whebe SCC, Kusumota L, Rodrigues RAP, Marques S. Fragilidade de idosos internados na clínica médica da unidade de emergência de um hospital geral terciário. Texto Contexto Enferm. 2013;22(2):452-9. doi: http://dx.doi. org/10.1590/S0104-07072013000200022. 